\title{
Clasificación de Gestos de la Lengua de Señas Colombiana a partir del Análisis de Señales Electromiográficas utilizando Redes Neuronales Artificiales
}

\author{
Elvis H. Galvis-Serrano ${ }^{1}$, Iván Sánchez-Galvis' ${ }^{1}$ Natalia Flórez ${ }^{1}$ y Sergio Zabala-Vargas ${ }^{1}$ \\ (1) Facultad de Ingeniería de Telecomunicaciones, Universidad Santo Tomás, Cr 18 \# 9-27, Bucaramanga- \\ Colombia (e-mail: elvis.galvis@ustabuca.edu.co; ivan.sanchez@ustabuca.edu.co; \\ yudy.florez@ustabuca.edu.co; sergio.zabala@ustabuca.edu.co).
}

Recibido Jul. 20, 2018; Aceptado Oct. 2, 2018; Versión final Nov. 16, 2018, Publicado Abr. 2019

\begin{abstract}
Resumen
El objetivo del presente trabajo es clasificar los 27 gestos del alfabeto de señas colombiano, mediante un clasificador de redes neuronales artificiales a partir de señales electromiográficas. El clasificador fue diseñado en cuatro fases: 1) Adquisición de señales electromiográficas provenientes de los ocho sensores de la manilla Myo Armband, 2) Extracción de características de las señales electromiográficas empleando la transformada Wavelet de Paquetes, 3) Entrenamiento de la red neuronal y 4) Validación del método de clasificación utilizando la técnica de validación cruzada. Para el presente estudio se adquirieron registros de señales electromiográficas de 13 sujetos con discapacidad auditiva. El clasificador presentó un porcentaje de precisión promedio de $88,4 \%$, muy similar a otros métodos de clasificación presentados en la literatura. El método de clasificación puede ser escalado para clasificar, adicional a los 27 gestos, el vocabulario de la lengua de señas colombiana.
\end{abstract}

Palabras clave: lengua de señas colombiana; Myo Armband; redes neuronales; validación cruzada; Wavelet

\section{Classification of Gestures of the Colombian Sign Language from the Analysis of Electromyographic signals using Artificial Neural Networks}

\begin{abstract}
The objective of this article is to classify the 27 gestures of the Colombian sign alphabet, by means of a classifier of artificial neural networks based on electromyographic signals. The classifier was designed in four phases: Acquisition of electromyographic signals from the eight sensors of the Myo Armband handle, extraction of characteristics of the electromyographic signals using the wavelet transform of packages, training of the neural network and validation of the classification method using the cross-validation technique. For the present study, records of electromyographic signals from 13 subjects with hearing impairment were acquired. The classifier presented an average accuracy percentage of $88.4 \%$, very similar to other classification methods presented in the literature. The classification method can be scaled to classify, in addition to the 27 gestures, the vocabulary of the Colombian sign language.
\end{abstract}

Keywords: Colombian sign language; Myo Armband; neural networks; cross validation; Wavelet 


\section{INTRODUCCIÓN}

La sordomudez, es la imposibilidad que tiene una persona de hablar y de oír. Este síndrome generalmente ocasionado por la existencia de una sordera congénita o adquirida en los primeros días o meses de vida causa que la persona no pueda reconocer sus propios sonidos y por lo tanto sea incapaz de hablar. Cerca de 466 millones de personas a nivel mundial padecen de pérdida de audición, y de esta cifra 34 millones son niños. Un ejercicio prospectivo establece que, hacia 2050, se proyecta que más de 900 millones de personas padecerán de esta discapacidad (World Health Organization, 2018). Las personas con este síndrome pueden comprometer seriamente el desarrollo emocional, cognitivo, social y económico. La falta de capacidad de comunicarse fácilmente con las demás personas puede causar situaciones que generen bajo rendimiento escolar, sensación de soledad, aislamientos, frustración, y desempleo (Better Hearing Institute, 2016). StangAlva (2012), cita en su publicación realizada para el Centro Latinoamericano y Caribeño de DemografíaCELADE, adscrito a la Comisión Económica para América Latina y el Caribe- CEPAL, que un alto porcentaje de las personas con discapacidad viven por debajo del umbral de pobreza en los países en desarrollo. A su vez, se encuentran evidencias que la participación de las personas con discapacidad en la fuerza laboral es muy inferior a las personas sin esta condición.

Para generar espacios de inclusión y dinamizar la forma de comunicación entre personas sordas y personas oyentes, la comunidad científica se ha interesado en realizar investigaciones que tienen como propósito el mejoramiento de la calidad de vida de las personas sordas, buscando facilitar la inserción en un contexto de comunicación con personas sin dificultades auditivas. La revisión de la literatura evidencia que las investigaciones están empleando diferentes dispositivos tecnológicos que permiten facilitar la comunicación entre las personas con discapacidad y aquellas que no la tienen, así como el estudio de herramientas tecnológicas como interfaz hombre-máquina. Entre los dispositivos se encuentran: 1) Cámaras de video e imágenes, basadas en diversos algoritmos de tratamiento de señales y extracción de características (Ciaramello y Hemami, 2011; Guerrero-Balaguera y Pérez-Holguín, 2015; Upendran y Thamizharasi, 2014); 2) MS Kinect como herramienta de registro de movimiento, fundamentado en diferentes estrategias de procesamiento de video y detección de movimiento (Ahmed et al., 2016; Jimenez et al., 2017; Sosa-Jiménez et al.,2017); 3) Leap Motion como herramienta para registro del movimiento y gestos de las manos, y las distancias entre los dedos (Heloir y Nunnari, 2016; Hisham y Hamouda, 2017; Quesada et al.,2017); 4) Guantes para registro de movimiento (Gałka et al.,2016; Sarawate et al., 2015) y 5) Dispositivo Thalmic Labs Myo Armband y su registro de señales mioeléctricas (Abreu et al.,2016; Akhmadeev et al., 2017; Wan et al., 2017).

Enfatizando en este último dispositivo (Myo Armband), se encuentra la experiencia presentada por Abreu et al. (2016) para la caracterización del lenguaje de señal brasilero (denominado por el acrónimo LIBRAS) y la experiencia presentada por Khamid et al. (2017) para el sistema de lengua de señas de Indonesia (ISLS). La primera experiencia realizó la clasificación de 20 letras estáticas del alfabeto por medio de máquinas binarias de soporte vectorial (SVM); en esta investigación no se avanzó en aquellas gestos que requieren movimiento, se priorizó los gestos estáticos; en la segunda experiencia se implementó un sistema que mejora el rendimiento y la precisión de clasificación al combinar los datos provenientes del Leap Motion, con los electrodos EMG del dispositivo Myo Armband, utilizando un clasificador probabilístico Naïve Bayes. En Tailandia emplearon electrodos similares a los de la manilla Myo para el registro de 10 gestos del lenguaje de señas tailandés, por medio de un modelo no lineal de redes neuronales se obtuvo una precisión de coincidencia con la salida de más del 95\% (Amatanon et al., 2014). En Sri Lanka se realizó una investigación que compara la exactitud de soluciones basadas en TI como: Kinect con un $90 \%$, Data Glove $80 \%$, Leap Motion del $95 \%$ al $98 \%$, procesamiento de imágenes $90 \%$, y dispositivos que combinan EMG con IMU como es el caso de la manilla Myo con un 98\% de exactitud (Khushaba et al., 2011). Si bien, la literatura ha presentado resultados de investigaciones orientadas a brindar apoyo a la discapacidad citada empleando diversas opciones tecnológicas, no se tiene conocimiento de investigaciones específicas con la lengua de señas colombiana empleando señales electromiográficas (EMG). El presente trabajo propone un clasificador de señales EMG utilizando redes neuronales artificiales para clasificar los 27 gestos del alfabeto de señas colombiano.

\section{METODOLOGÍA}

Para diseñar el clasificador de gestos del alfabeto de señas colombiano se definieron cuatro fases: 1) Adquisición de las señales EMG provenientes de los ocho sensores, registradas por la manilla Myo Armband, 2) extracción de características de las señales empleando la transformada Wavelet, 3) entrenamiento de la red neuronal y 4) validación del método de clasificación utilizando la técnica de validación cruzada. Las diferentes fases fueron soportadas en la herramienta software Matlab® de Mathworks. Para el presente estudio se adquirieron registros de señales EMG en 13 sujetos con discapacidad auditiva: 4 sujetos entre 10 y 20 años, 6 sujetos entre 21 y 40 años y 3 sujetos mayores de 40 años. Los sujetos son pertenecientes a la asociación de Sordos de Santander ASORSAN en Bucaramanga y a la fundación para la inclusión laboral 
educativa y social del Magdalena Medio- ILESMAG en Barrancabermeja, todos los participantes fueron informados de la investigación y firmaron el respectivo consentimiento informado.

\section{Fase de Adquisición de señales electromiográficas}

La adquisición de las señales EMG es realizada empleando la manilla Myo Armband, la cual es un dispositivo de reconocimiento de gestos que se ubica en el antebrazo y registra a través de ocho sensores, la actividad eléctrica generada en la contracción muscular. El dispositivo cuenta también con giróscopo, acelerómetro, orientación (IMU), así como comunicación inalámbrica por bluetooth para emparejarse con un equipo de cómputo. Las señales EMG son adquiridas a una frecuencia de muestreo de $200 \mathrm{~Hz}$ y las asociadas a la IMU a $50 \mathrm{~Hz}$. El firmware que acompaña la manilla permite generar una interfaz de usuario propietaria, así como el diseño de protocolos de calibración por individuo; lo cual es muy útil para mejorar la precisión del dispositivo. Al realizar la adquisición de un gesto del alfabeto de señas colombiano con la manilla Myo Armband se obtiene 8 señales EMG como se observa en la figura 1.

Por cada uno de los 13 sujetos, se adquirieron las 8 señales EMG de cada uno de los 27 gestos del alfabeto de señas colombiano. Para cada uno de los gestos la adquisición tuvo una duración de 5 segundos, con una frecuencia de muestreo de $200 \mathrm{~Hz}$, obteniéndose un total de 1000 muestras de cada letra por sujeto. Los datos de cada letra fueron organizados en matrices de 1000 filas $\times 8$ columnas, donde cada columna representa una señal EMG y las filas muestras.

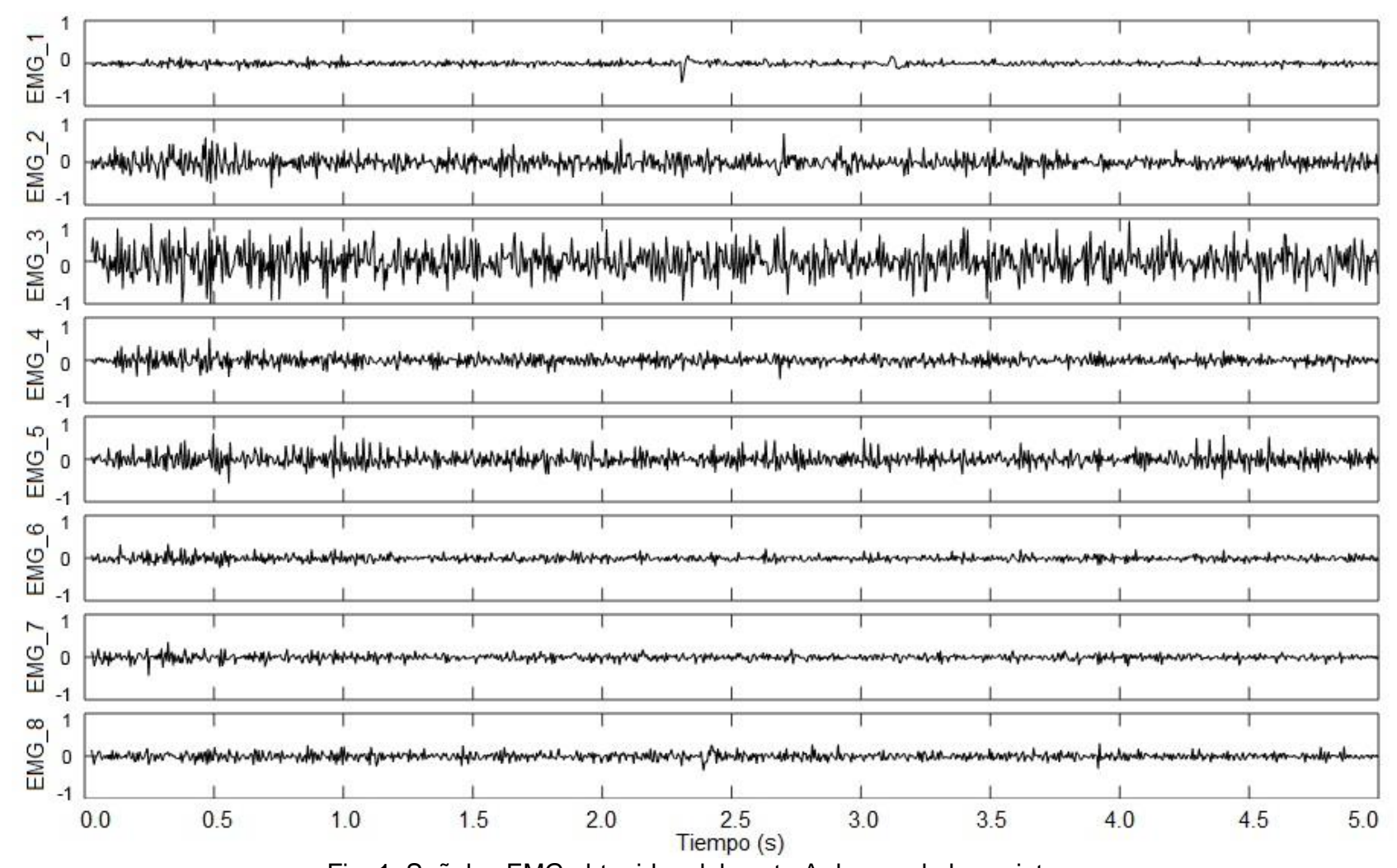

Fig. 1: Señales EMG obtenidas del gesto $A$ de uno de los sujetos.

Fase de Extracción de Características

Las señales de origen biológico, entre las cuales se encuentran las señales EMG, tienen un comportamiento estocástico no estacionario. El procesamiento de señales con la técnica de análisis espectral basada en la Transformada Wavelet ha sido empleado ampliamente en el procesamiento de señales no estacionarias en diferentes aplicaciones (González-Acevedo et al.,2015; Khushaba et al., 2011; Sanabria-Sarmiento y ZabalaVargas, 2010). La extracción de las características de la señal EMG $x_{i}(t)(i=1, \ldots, 8)$ se realizó empleando la Transformada de Paquetes Wavelet (Wavelet Packet Transform- WPT), introducida por (Coifman et al, 1992). En la WPT, la señal $x_{i}(t)$ se descompone a partir de dos bases ortogonales $\varphi^{j, k}(t)$ (Función de aproximación) y $\psi^{j, k}(t)$ (Función de detalle), donde $j=1, \ldots, J$ indica la escala, $J$ es el máximo nivel de descomposición y $k$ es el indice de la sub-banda dentro de la escala. La diferencia con la transformada wavelet es que, para los niveles de descomposición posteriores, el WPT no solo descompone los coeficientes de aproximación, sino que también los coeficientes de detalle. La Figura 2 muestra el proceso de descomposición con la WPT. 


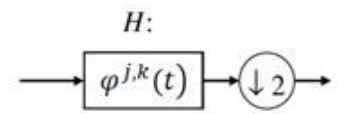

Filtro pasabajas y diezmado

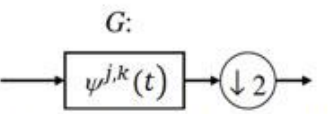

Filtro pasaltas y diezmado

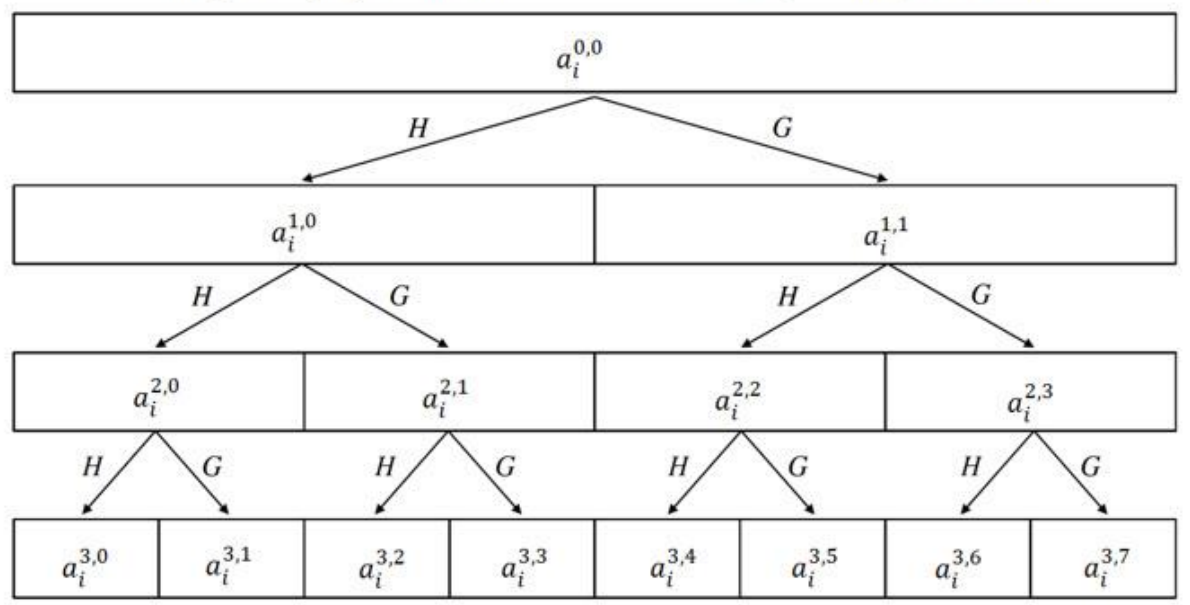

Fig. 2: Descomposición WPT en coeficientes de aproximación y detalle hasta el nivel 3. Esta descomposición debe hacerse para cada uno de los canales de la señal electromiográfica. (Adaptado de (Wu \& Liu, 2009))

Los coeficientes de aproximación y detalle de la señal $x_{i}(t)$ se obtienen con la ecuación 1

$a_{i}^{j, k}=\left\langle x_{i}(t), \varphi^{j, k}(t)\right\rangle, y d_{i}^{j, k}=\left\langle x_{i}(t), \psi^{j, k}(t)\right\rangle$

Donde $\langle$,$\rangle indica el producto interno.$

Cada uno de los coeficientes de aproximación y detalle son tomados como una característica para el algoritmo de clasificación. En el presente estudio se emplearon 8 señales EMG y se tomaron los coeficientes de aproximación y detalle del nivel de descomposición 3 (Khushaba et al, 2011) y el nivel de descomposición 4 (Phinyomark et al., 2009). Esto da como resultado $2^{3}+2^{4}=24$ características por señal EMG, obteniendo $24 \times 8=192$ características en total.

La descomposición se realizó con una Wavelet Daubechies 4 con ventana de 40 muestras y solapamiento del $75 \%$. De acuerdo con la relación dada en la ecuación 2, a partir de las 1000 muestras de cada señal EMG se obtendrían en total 97 ventanas (ejemplos). Los datos de cada letra se organizan en matrices de 97 filas por 192 columnas, donde las filas corresponden al número de ejemplos y cada 24 columnas corresponde a las características de cada señal EMG, obteniendo al final un total de 192 características por las 8 señales EMG (Figura 3).

$$
m=\frac{N-V}{(1-s) V}
$$

Donde $m$ es el número de ejemplos, $N$ es el número de muestras de cada señal EMG, $V$ es la longitud de la ventana en muestras y $s$ es el porcentaje de solapamiento del proceso de enventanado.
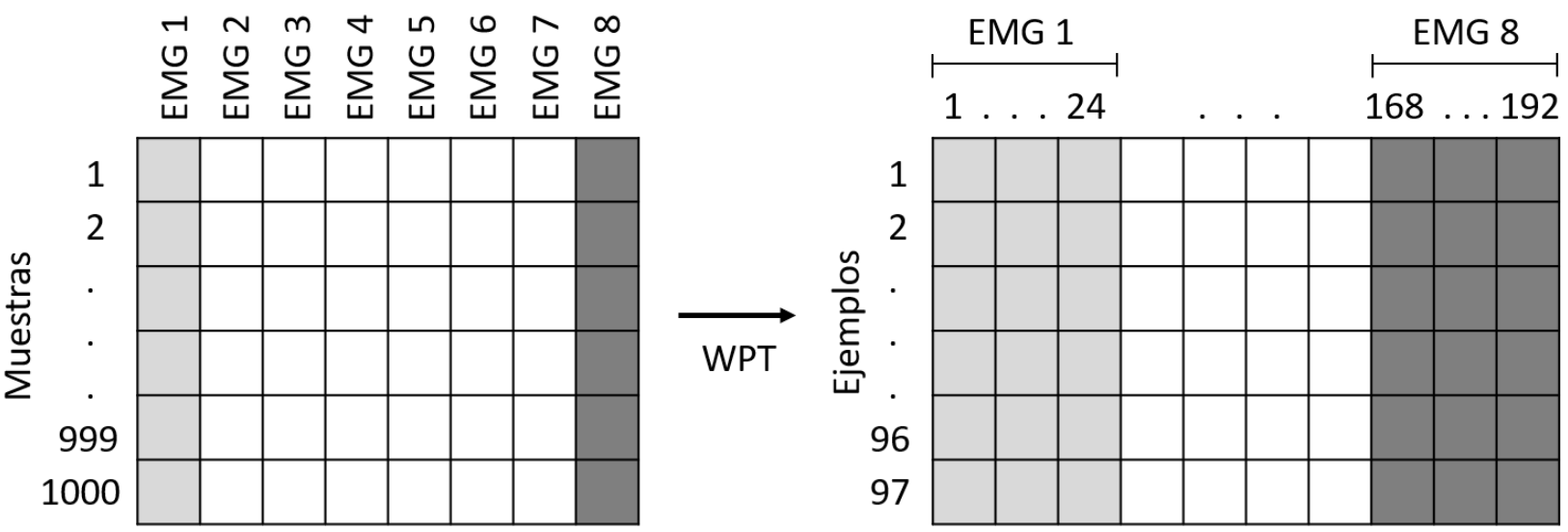

Fig. 3: Matriz de características de la señal $A$ 


\section{Fase de entrenamiento}

Existen diferentes métodos de clasificación de señales EMG. En Dellacasa et al. (2017), compararon cuatro clasificadores: Nonlinear Logistic Regression (NLR), Linear Discriminant Analysis (LDA), Multi-Layer Perceptron (MLP, el cual es un tipo de Red Neuronal) y Support Vector Machine (SVM); los resultados indicaron que los dos últimos mencionados fueron los de mayor precisión (Scheme y Englehart, 2011). En la presente investigación se empleó Redes Neuronales Artificiales (ANN, Artificial Neural Networks) en lugar de SVM debido a que esta última es usada para clasificación de datos en exactamente dos clases. A pesar de que SVM también puede adaptarse para clasificación multiclase, dicha adaptación podría resultar en un clasificador que se ajuste muy bien a los datos de entrenamiento, pero cuya precisión sea baja ante nuevos patrones de entrada, esto debido a la alta sensibilidad a pequeños cambios en posición y fuerza aplicada al momento de realizar el gesto con la mano (Abreu et al, 2016).

Las ANNs son un algoritmo de aprendizaje que permite crear modelos para la clasificación de datos a partir de ejemplos de entrenamiento y existen múltiples tipos, los cuales se detallan en (Mateus et al., 2014). En general, las ANNs son un sistema de ecuaciones no lineales cuyos parámetros son ajustados por medio de un algoritmo de retropropagación (Backpropagation Algorithm) que minimiza el error entre los datos de entrenamiento y los datos modelados por la red neuronal. Las redes neuronales constan de tres o más capas: una capa de entrada, una o más capas ocultas y una capa de salida. Cada una de estas capas se modelan siguiendo las ecuaciones 3,4 y 5 .

Capa de entrada: $\mathbf{x}^{(i)}$

Capa oculta: $\mathbf{a}_{1}^{(i)}=\sigma\left(\mathbf{W}_{1} \mathbf{x}^{(i)}+\mathbf{b}_{1}\right)$

Capa de salida: $\hat{\mathbf{y}}^{(i)}=\sigma\left(\mathbf{W}_{2} \mathbf{a}_{1}^{(i)}+\mathbf{b}_{2}\right)$

Donde $\mathbf{x}^{(i)}$ es el ejemplo $i$ de los datos de entrada; $\mathbf{W}_{1}, \mathbf{b}_{1}, \mathbf{W}_{2}$ y $\mathbf{b}_{2}$ son las matrices de pesos y los vectores de términos independientes de las capas 1 y 2 , respectivamente; y $\sigma$ es la función de activación no lineal. El resultado de la capa de salida $\hat{\mathbf{y}}^{(i)}$ es la estimación $i$ de la salida deseada $\mathbf{y}^{(i)}$. Los pesos se inicializan aleatoriamente y los términos independientes comienzan en cero. Durante el entrenamiento, los pesos y los términos independientes se actualizan de manera iterativa para asegurar que la red converge en una aproximación óptima a la salida esperada.

Una vez se obtienen las 27 matrices de características, éstas son apiladas conformando una única matriz de 2619 filas x 192 columnas, donde las filas corresponden a los 97 ejemplos de los 27 gestos y las columnas las características (Figura 4a). Para etiquetar los datos, se creó una matriz de 2619 filas (ejemplos) x 27 columnas (clases) en donde cada fila representa el vector de salida deseado $\mathbf{y}=\left[y_{1}, \ldots, y_{27}\right]$, el cual se define en la ecuación 6 (Figura 4b).

$$
y_{i}=\left\{\begin{array}{c}
1, \text { si } i=j ; \text { donde } j \text { es la clase } \\
0, \text { para otros valores }
\end{array}\right.
$$
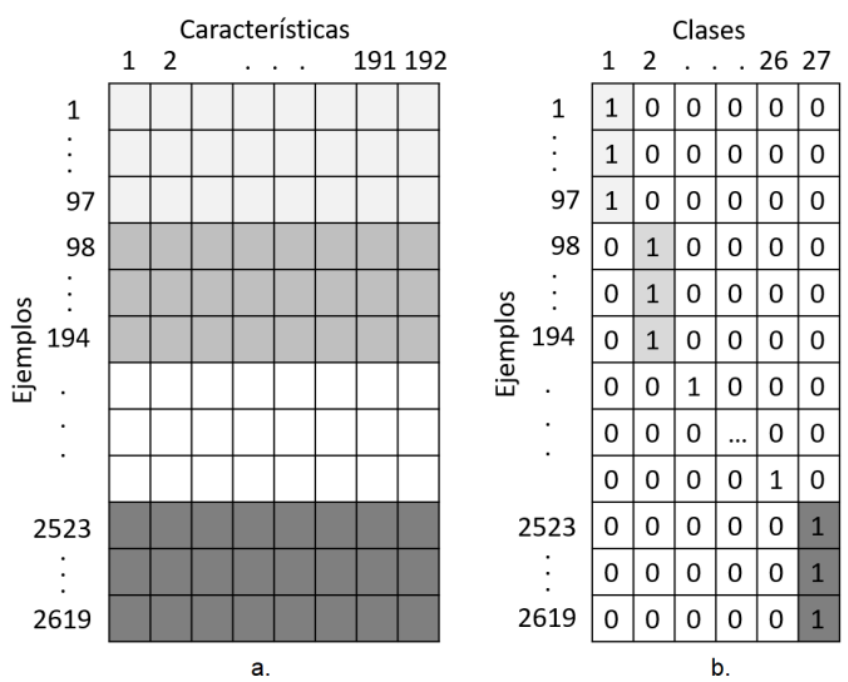

Fig. 4: a) Matriz de características. b) Matriz de clases 
Se entrenó una red neuronal supervisada del tipo Perceptrón Multicapa (MLP) empleando la herramienta nprtool de Matlab. El número de capas seleccionado fue 40 . Se utilizaron las funciones de activación sigmoide y softmax para la capa oculta y de salida, respectivamente (Figura 5). Los pesos y los términos independientes fueron actualizados con el algoritmo de retropropagación de gradiente conjugado escalado. La elección de este tipo de red y el número de capas se argumenta en estudios donde se demuestra su óptimo rendimiento ante otras técnicas de reconocimiento para señales EMG (Elamvazuthi et al., 2015) y mayor índice de reconocimiento empleando múltiples características de la señal (Hamedi et al., 2014).

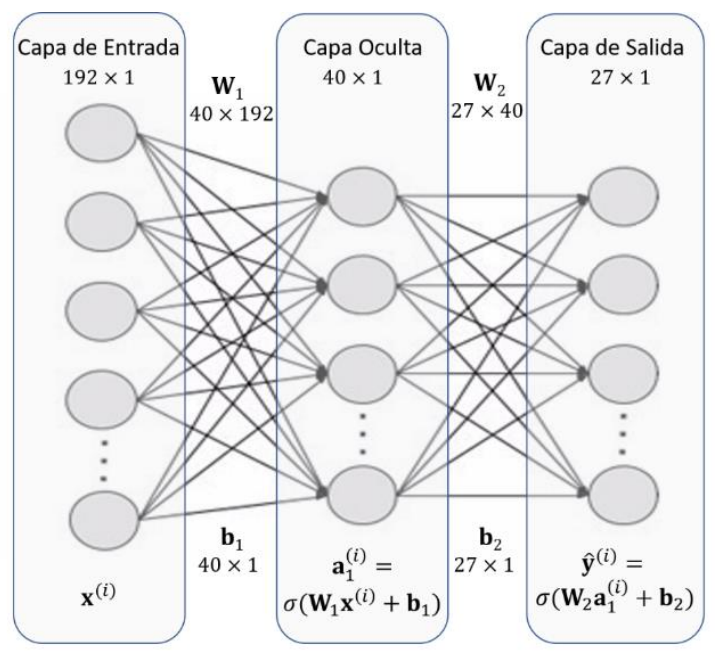

Fig. 5: Estructura específica de la red neuronal.

\section{Fase de Validación}

Cross-validation o validación cruzada es una técnica ampliamente utilizada en proyectos de inteligencia artificial, y sirve para evaluar los resultados de análisis estadísticos y garantizar la independencia entre datos de entrenamiento y prueba de modelos generados. Es utilizada en proyectos en donde se quiere estimar la precisión de un modelo que se llevará a cabo a la práctica (Forman y Scholz, 2010). Con el propósito de evaluar la precisión del método de clasificación realizado mediante el entrenamiento de la red neuronal se diseñó una etapa de Crossvalidation, empleada en la función crossvalind de Matlab, con factor de iteración $\mathrm{K}=10$, factor más empleado en la literatura revisada (Abreu et al., 2016; Kari et al., 2018; Zhang et al., 2015).

La red neuronal fue entrenada con el $90 \%$ de las muestras de la matriz transpuestas de características y supervisada con la matriz transpuesta de unos y ceros generadas a la salida del proceso de la Wavelet. El 10\% de las muestras restantes fueron empleadas para la validación. Esto se realiza 10 veces, para cada iteración de $\mathrm{k}$, con distintas muestras de entrenamiento y validación, obteniendo en cada iteración un coeficiente de precisión. Posteriormente se calculó el valor promedio y desviación estándar de los valores de coeficientes de precisión obtenidos en las 10 interacciones.

\section{RESULTADOS Y DISCUSIÓN}

Se presenta el cómo se conformó con base de datos, la clasificación de ésta, y las características y composición de los grupos en estudio.

\section{Base de datos de señales}

Se creó una base de datos con los registros de las 27 letras del alfabeto colombiano de 13 personas sordas. Las personas fueron clasificadas en 3 grupos dependiendo de la edad. El número de registros realizados por edades se presenta en la Tabla 1.

Tabla 1: Registros por edades.

\begin{tabular}{|c|c|c|}
\hline$\#$ & Edades & $\begin{array}{c}\text { Registros } \\
\text { realizados }\end{array}$ \\
\hline Grupo 1 & 10-20 años & 4 \\
\hline Grupo 2 & 21-40 años & 6 \\
\hline Grupo 3 & + de 40 años & 3 \\
\hline & Total & 13 \\
\hline
\end{tabular}




\section{Precisión del clasificador}

Los 13 sujetos participantes en la investigación fueron clasificados en tres grupos por rango de edad para calcular la precisión del método de clasificación. Cada registro se compone de la adquisición de las ocho señales EMG originadas al realizar cada uno de los 27 gestos del alfabeto. En la Tabla 2 se presenta, el rango de edad, el número de registros, los valores de precisión del método de clasificación: máximo, mínimo, promedio y la desviación estándar en cada uno de los grupos. Los valores de precisión máximo, mínimo, promedio y la desviación estándar de todos los registros, sin ser agrupados por edad son presentados en la Tabla 3.

Tabla 2: Precisión del método de caracterización en cada grupo

\begin{tabular}{|l|c|c|c|}
\hline & Grupo 1 & Grupo 2 & Grupo 3 \\
\hline Rango de edad & $10-20$ años & 21 a 40 años & $>40$ años \\
\hline Número de registros & 4 & 6 & 3 \\
\hline $\begin{array}{l}\text { Valor máx. de } \\
\text { precisión }\end{array}$ & 97,78 & 94,58 & 95,65 \\
\hline Valor min de precisión & 82,17 & 67,31 & 68,97 \\
\hline Promedio de precisión & 91,05 & 87,85 & 85,74 \\
\hline Desviación estándar & 3,47 & 7,86 & 8,95 \\
\hline
\end{tabular}

Tabla 3: Precisión del método de caracterización en la totalidad de los registros

\begin{tabular}{|l|c|}
\hline \multicolumn{2}{|c|}{ Precisión Total } \\
\hline Valor máx. de precisión & 97,78 \\
\hline Valor min de precisión & 67,31 \\
\hline Promedio de precisión & 88,39 \\
\hline Desviación estándar & 7,29 \\
\hline
\end{tabular}

El modelo de clasificación fue validado cuantitativamente por medio de la técnica Cross-validation, reflejando una precisión promedio del $88.39 \%$ (Tabla 3). La Figura 6 presenta el comportamiento de la precisión durante las 10 iteraciones, se relaciona por cada iteración, valores mínimos, máximos y la mayor concentración de datos. Por grupos de edades, los tres grupos presentaron valores de precisión máximos similares pero el grupo de mayor edad presentó una menor precisión y una mayor desviación estándar, atribuibles posiblemente al movimiento en la adquisición de las señales EMG por dificultad en la adaptación con la manilla Myo. Cabe destacar que el grupo más joven presentó la mayor precisión y desviación estándar más baja, posiblemente debido a la fácil adaptación con las nuevas tecnologías.

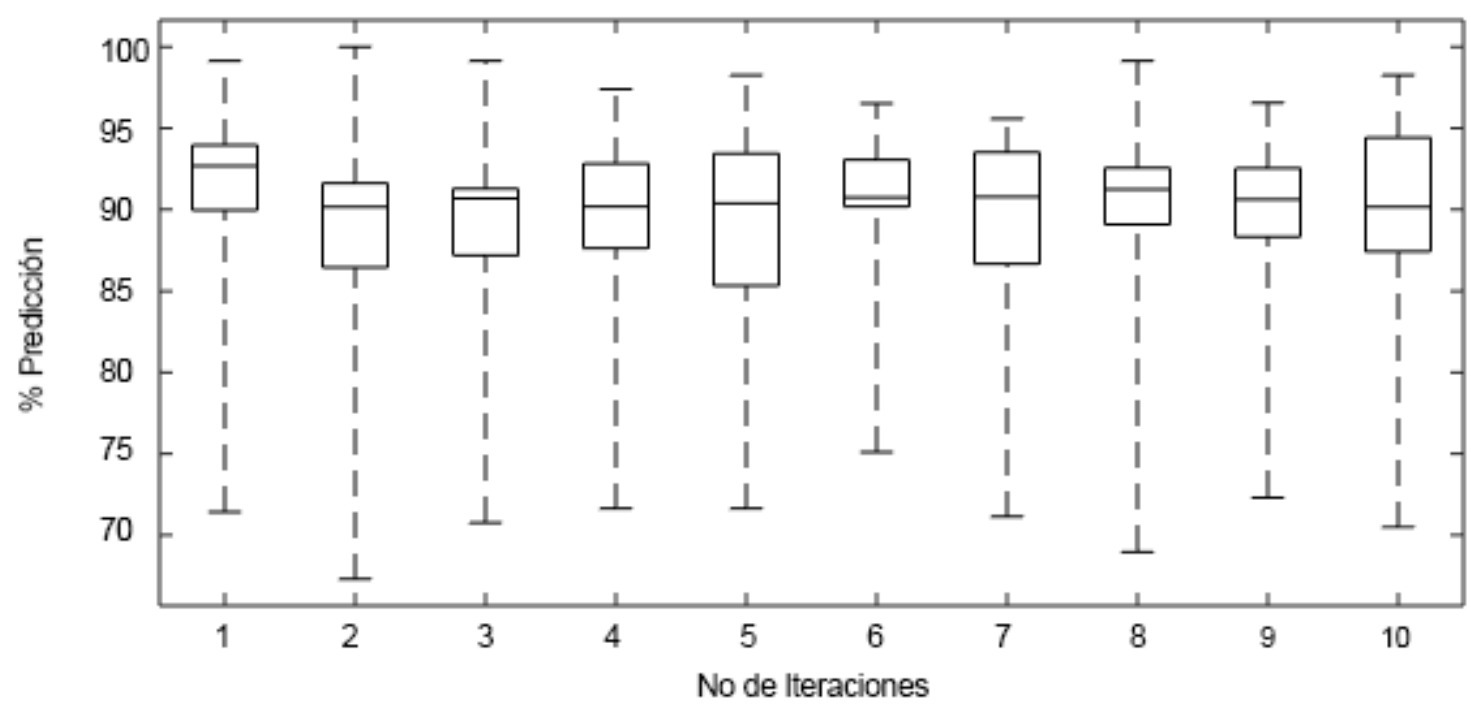

Fig. 6: Validación cruzada de la precisión del clasificador. 
El valor de precisión máxima obtenido (97,78\%) es similar al presentado por (Abreu et al., 2016) en sus resultados en tiempo real (95\%) empleando SVM, no obstante, los valores de precisión mínima obtenidos son muy alejados; el resultado obtenido en la presente investigación de mínima precisión es $67,31 \%$ mientras que el valor obtenido por (Abreu et al., 2016) es del 4\%. El estudio realizado por (Upendra y Thamizharasi, 2014) detalla la precisión obtenida en cada una de las clases del alfabeto de señas americano, si bien en la presente investigación no se presentan resultados por las diferentes clases del alfabeto de señas colombiano, se comparan los resultados del rango y valor promedio de precisión obtenidos teniendo en cuenta la similitud entre los dos alfabetos. Los resultados de (Upendra y Thamizharasi, 2014) empleando un sistema PCA-KNN presentan una variación más amplia de precisión, la cual varía entre $0 \%$ y $100 \%$ con una precisión promedio de $77,30 \%$, mientras que la variación de precisión obtenida en el estudio del alfabeto de señas colombiano se encuentra entre el $67,31 \%$ y $97,78 \%$ con un valor promedio de $88,39 \%$.

El promedio de precisión del sistema propuesto (88.39\%) se encuentra muy cercano al basado en LSTM (Long Short-Term memory) presentado por (Liu et al.,2016), cuya precisión máxima fue de $86.23 \%$. Estos resultados superan a la precisión obtenida con otras estrategias, como el método HMM (Normal Hidden Markov Models) y el método CM VoM (Curve Matching from the View of Manifold), citadas en este mismo trabajo. Al comparar el resultado de precisión máxima obtenida en el experimento (97.7\%) con el presentado por (Khushaba, Kodagoda, Lal, y Dissanayake, 2011), con una precisión del 98\%, y el mostrado por (Gałka et al., 2016), de $99.7 \%$, se observa una alta cercanía en el resultado. Al revisar los aspectos cualitativos citados en otras experiencias de la literatura se resalta la importancia de continuar la investigación mejorando las estrategias de calibración de la manilla MYO Armband en cada uno de los pacientes y la optimización del algoritmo de procesamiento.

\section{CONCLUSIONES}

De los resultados obtenidos y analizados, se pueden obtener las siguientes conclusiones: 1) Se cuenta con un clasificador de redes neuronales artificiales a partir de señales electromiográficas que permite clasificar los 27 gestos del alfabeto de señas colombiano con una precisión del $88.39 \%$. Este clasificador, con pruebas iniciales en ambiente real para la población con discapacidad oyente o hablante en Colombia, es la fase experimental para el desarrollo de una herramienta tecnológica a futuro. 2) El porcentaje de precisión del clasificador obtenido por rangos de edades o de manera global presenta un porcentaje muy similar a otras aproximaciones tecnológicas presentadas en la literatura, lo que permite contrastar la validez de la propuesta desarrollada; 3) La metodología propuesta puede ser escalada para clasificar adicional a los 27 gestos, el vocabulario del lenguaje de señas colombiano. 4) De acuerdo con el análisis de resultados, el clasificador presentó un porcentaje de precisión promedio cercano a los alcanzados por otros métodos mostrados en la literatura.

\section{REFERENCIAS}

Abreu, J.G., J. M. Teixeira, L. S. Figueiredo y V. Teichrieb, Evaluating Sign Language Recognition Using the Myo Armband, XVIII Symposium on Virtual and Augmented Reality (SVR), 64-70, Gramado-Brasil, 21-24 de Junio (2016)

Ahmed, M., M. Idrees, Z. Abideen, R. Mumtaz y S. Khalique, Deaf talk using 3D animated sign language: A sign language interpreter using Microsoft's Kinect v2, SAI Computing Conference (SAI), 330-335, Londres-Inglaterra, 13-15 de Julio (2016)

Akhmadeev, K, E. Rampone, T. Yu, Y. Aoustin y E. Carpentier, A testing system for a real-time gesture classification using surface EMG, IFAC-Papers Online, 50(1), 11498-11503 (2017)

Amatanon, V., S. Chanhang, P. Naiyanetr y S. Thongpang, Sign language-Thai alphabet conversion based on Electromyogram (EMG), 7th 2014 Biomedical Engineering International Conference, 1-4, Fukuoka-Japón, 27-28 de Noviembre (2014)

Better Hearing Institute, Consequences of Hearing Loss, BHI (2016)

Ciaramello, F.M. y S.S. Hemami, A Computational Intelligibility Model for Assessment and Compression of American Sign Language Video, doi: 10.1109/TIP.2011.2132730, IEEE Transactions on Image Processing, 20(11), 3014-3027 (2011)

Dellacasa, A., E. Gruppioni, G. Colazzo, A. Davalli, R. Sacchetti, E. Guglielmelli y L. Zollo, NLR, MLP, SVM, and LDA: a comparative analysis on EMG data from people with trans-radial amputation, doi: 0.1186/s12984-017-0290-6, Journal of Neuroengineering and Rehabilitation, 14(1), 82-93 (2017)

Elamvazuthi, I., N.H.X Duy, Z. Ali, S.W. Su, A. Khan y S. Parasuraman, Electromyography (EMG) based Classification of Neuromuscular Disorders using Multi-Layer Perceptron, doi: 10.1016/j.procs.2015.12.346, Procedia Computer Science, 76, 223-228 (2015)

Forman, G. y M. Scholz, Apples-to-apples in Cross-validation Studies: Pitfalls in Classifier Performance Measurement, doi: 10.1145/1882471.1882479, SIGKDD Explor. Newsl., 12(1), 49-57 (2010)

Gałka, J., M. Mąsior, M. Zaborski y K. Barczewska, Inertial Motion Sensing Glove for Sign Language Gesture Acquisition and Recognition, doi: 10.1109/JSEN.2016.2583542, IEEE Sensors Journal, 16(16), 6310-6316 (2016) 
González-Acevedo, H., C. J. Arizmendi-Pereira y B. Giraldo-Giraldo, Diseño de un clasificador para pacientes en proceso de extubación, doi:10.15332/iteckne.v12i2.1239, Revista Iteckne, 12(1), 131-137 (2015)

Guerrero-Balaguera, J. D. y W.J. Pérez-Holguín, FPGA-based translation system from Colombian sign language to text, doi: 10.15446/dyna.v82n189.43075, Revista DYNA, 82(189), 172-181(2015)

Hamedi, M., S. Salleh, K. Ismail, A.M. Noor, M. Astaraki y H. Aslian, Time-frequency facial gestures EMG analysis using bilinear distribution, 2015 IEEE International Conference on Signal and Image Processing Applications (ICSIPA), 169-173, Kuala Lumpur-Malasya, 19-21 de Octubre (2015)

Heloir, A. y F. Nunnari, Toward an intuitive sign language animation authoring system for the deaf, doi: 10.1007/s10209015-0409-0, Universal Access in the Information Society, 15(4), 513-523 (2016)

Hisham, B. y A. Hamouda, Arabic static and dynamic gestures recognition using leap Motion, doi: 10.3844/jcssp.2017.337.354, Journal of Computer Science, 13(8), 337-354 (2017)

Jimenez, J., A. Martin, V. Uc y A. Espinosa, Mexican Sign Language Alphanumerical Gestures Recognition using 3D Haarlike Features, doi: 10.1109/TLA.2017.8071247, IEEE Latin America Transactions, 15(10), 2000-2005 (2017)

Kari, T., W. Gao, D. Zhao, Z. Zhang, W. Mo, Y. Wang y L. Luan, An integrated method of ANFIS and Dempster-Shafer theory for fault diagnosis of power transformer, doi: 10.1109/TDEI.2018.006746, IEEE Transactions on Dielectrics and Electrical Insulation, 25(1), 360-371 (2018)

Khamid, A.D. Wibawa y S. Sumpeno, Gesture Recognition for Indonesian Sign Language Systems (ISLS) Using Multimodal Sensor Leap Motion and Myo Armband Controllers Based-on Naïve Bayes Classifier, 2017 International Conference on Soft Computing, Intelligent System and Information Technology (ICSIIT), 1-6, Bali-Indonesia,12-15 de Julio (2017)

Khushaba, R. N., S. Kodagoda, S. Lal, Dissanayake, Driver Drowsiness Classification Using Fuzzy Wavelet-Packet-Based Feature-Extraction Algorithm, doi: 10.1109/TBME.2010.2077291, IEEE Transactions on Biomedical Engineering, 58(1), 121-131 (2011)

Liu, T., W. Zhou y H. Li, Sign language recognition with long short-term memory, 2016 IEEE International Conference on Image Processing (ICIP), 2871-2875, Phoenix-USA, Septiembre 25-28 de (2016)

Mateus, S. P., N. González y J.W. Branch, Aplicación de Redes Neuronales Artificiales en Entornos Virtuales Inteligentes, doi: 10.4067/S0718-07642014000500015, Información Tecnológica, 25(5), 103-112 (2014)

Phinyomark, A., C. Limsakul y P. Phukpattaranont, A Novel Feature Extraction for Robust EMG Pattern Recognition, Journal of Computing, 1(1), 71-80 (2009)

Quesada, L., G. López y L. Guerrero, Automatic recognition of the American Sign Language fingerspelling alphabet to assist people living with speech or hearing impairments, doi: 10.1007/s12652-017-0475-7, Journal of Ambient Intelligence and Humanized Computing, 8(4), 625-635 (2017)

Sanabria-Sarmiento, J.J. y S. Zabala-Vargas,Metodología para la determinación de usos del suelo mediante procesamiento de imágenes satelitales, doi:10.15332/iteckne.v7i1.357, ITECKNE, 7(1), 98-107 (2010)

Sarawate, N., M. Leu y C. Oz, A real-time American Sign Language word recognition system based on neural networks and a probabilistic model, doi: 10.3906/elk-1303-167, Turkish Journal Of Electrical Engineering \& Computer Sciences, 23, 2107-2123 (2015)

Scheme, E. y K. Englehart, Electromyogram pattern recognition for control of powered upper-limb prostheses: state of the art and challenges for clinical use, doi: 10.1682/JRRD.2010.09.0177, J. Rehabil Res, 48(6), 643-659 (2011)

Sosa-Jiménez, C.O., H. Ríos-Figueroa, E.J. Rechy-Ramírez, A. Marin-Hernandez y A. L. S. González-Cosío, Real-time Mexican Sign Language recognition, 2017 IEEE International Autumn Meeting on Power, Electronics and Computing (ROPEC),1-6, Ixtapa-México, 8-10 de Noviembre (2017)

Stang-Alva, M. F., Las personas con discapacidad en América Latina: del reconocimiento jurídico a la desigualdad real, CEPAL (2012)

Upendran, S. y A. Thamizharasi, American Sign Language interpreter system for deaf and dumb individuals, 2014 International Conference on Control, Instrumentation, Communication and Computational Technologies (ICCICCT), 14771481, 10-11 de Julio (2014)

Wan, B., R. Wu, K. Zhang y L. Liu, A new subtle hand gestures recognition algorithm based on EMG and FSR. 2017 IEEE 21st International Conference on Computer Supported Cooperative Work in Design (CSCWD), 127-132, WellingtonNueva Zelanda, 26-28 de Abril (2017)

World Health Organization, Deafness and hearing loss, WHO (2018)

Zhang, X., B. Hu, X. Ma y L. Xu, Resting-State Whole-Brain Functional Connectivity Networks for MCI Classification Using L2-Regularized Logistic Regression, doi: 10.1109/TNB.2015.2403274, IEEE Transactions on NanoBioscience, 14(2), 237$247(2015)$ 
